\title{
UNIFORM CONVERGENCE OF ERGODIC LIMITS AND APPROXIMATE SOLUTIONS
}

\author{
SEN-YEN SHAW
}

(Communicated by Paul S. Muhly)

\begin{abstract}
Let $A$ be a densely defined closed (linear) operator, and $\left\{A_{\alpha}\right\}$, $\left\{B_{\alpha}\right\}$ be two nets of bounded operators on a Banach space $X$ such that $\left\|A_{\alpha}\right\|=$ $O(1), A_{\alpha} A \subset A A_{\alpha},\left\|A A_{\alpha}\right\|=O(1)$, and $B_{\alpha} A \subset A B_{\alpha}=I-A_{\alpha}$. Denote the domain, range, and null space of an operator $T$ by $D(T), R(T)$, and $N(T)$, respectively, and let $P$ (resp. $B$ ) be the operator defined by $P x=\lim _{\alpha} A_{\alpha} x$ (resp. $B y=\lim _{\alpha} B_{\alpha} y$ ) for all those $x \in X$ (resp. $y \in \overline{R(A)}$ ) for which the limit exists. It is shown in a previous paper that $D(P)=N(A) \oplus \overline{R(A)}$, $R(P)=N(A), D(B)=A(D(A) \cap \overline{R(A)}), R(B)=D(A) \cap \overline{R(A)}$, and that $B$ sends each $y \in D(B)$ to the unique solution of $A x=y$ in $\overline{R(A)}$. In this paper, we prove that $D(P)=X$ and $\left\|A_{\alpha}-P\right\| \rightarrow 0$ if and only if $\left\|B_{\alpha} \mid D(B)-B\right\| \rightarrow 0$, if and only if $\left\|B_{\alpha} \mid D(B)\right\|=O(1)$, if and only if $R(A)$ is closed. Moreover, when $X$ is a Grothendieck space with the Dunford-Pettis property, all these conditions are equivalent to the mere condition that $D(P)=X$. The general result is then used to deduce uniform ergodic theorems for $n$-times integrated semigroups, $(Y)$-semigroups, and cosine operator functions.
\end{abstract}

\section{INTRODUCTION}

Let $X$ be a Banach space and $B(X)$ be the set of all bounded linear operators on $X$. Let $A: D(A) \subset X \rightarrow X$ be a densely defined closed linear operator, and let $\left\{A_{\alpha}\right\}$ and $\left\{B_{\alpha}\right\}$ be two nets in $B(X)$ satisfying the conditions:

(C1) $\left\|A_{\alpha}\right\|=O(1)$, i.e., there exist $M$ and $\alpha_{0}$ such that $\left\|A_{\alpha}\right\| \leq M$ for $\alpha \geq \alpha_{0}$

(C2) $R\left(B_{\alpha}\right) \subset D(A)$ and $B_{\alpha} A \subset A B_{\alpha}=I-A_{\alpha}$ for all $\alpha$,

(C3) $R\left(A_{\alpha}\right) \subset D(A)$ and $A_{\alpha} A \subset A A_{\alpha}$, and $\left\|A A_{\alpha}\right\| \rightarrow 0$.

These two systems of operators have been employed in our earlier papers [13] and [14] to formulate an abstract mean ergodic theorem and to produce approximate solutions of the functional equation $\mathrm{Ax}=y$.

Let $P$ be the operator defined by $P x:=s-\lim _{\alpha} A_{\alpha} x$ for $x \in D(P):=$ $\left\{x \in X ; s-\lim _{\alpha} A_{\alpha} x\right.$ exists $\}$, and let $B$ be the operator defined by $B y:=$ $s-\lim _{\alpha} B_{\alpha} y$ for $y \in D(B):=\left\{y \in \overline{R(A)} ; s-\lim _{\alpha} B_{\alpha} y\right.$ exists $\}$. The following two

Received by the editors July 23, 1990.

1980 Mathematics Subject Classification (1985 Revision). Primary 47A35, 47A50, 47D05.

Key words and phrases. Uniform ergodicity, linear functional equation, Grothendieck space with the Dunford-Pettis property, $n$-times integrated semigroup, cosine operator function.

This research was supported in part by the National Science Council of the R.O.C. 
strong convergence theorems were proved in [13]: (i) $P$ is a bounded linear projection with range $R(P)=N(A)$, null space $N(P)=\overline{R(A)}$, and domain $D(P)=N(A) \oplus \overline{R(A)}=\left\{x \in X ;\left\{A_{\alpha} x\right\}\right.$ has a weak cluster point $\}$; (ii) $B$ is the inverse operator of the restriction $A \mid \overline{R(A)}$ of $A$ to $\overline{R(A)}$; it has range $R(B)=D(A) \cap \overline{R(A)}$ and domain $D(B)=A(D(A) \cap \overline{R(A)})=\left\{y \in \overline{R(A)} ;\left\{B_{\alpha} y\right\}\right.$ has a weak cluster point $\}$. Moreover, for any given $y \in D(B)$, the vector $B y$ is the unique solution of the functional equation $\mathrm{Ax}=y$ that lies in $\overline{R(A)}$. This closed operator $B$ is called the inner inverse of $A$.

Actually, the convergence of $B_{\alpha} y$ to $B y=(A \mid \overline{R(A)})^{-1} y$ for $y \in D(B)$ is seen from the following computation using (C2) and (i).

$$
\begin{aligned}
\left\|B_{\alpha} y-B y\right\| & =\left\|B_{\alpha} A B y-B y\right\|=\left\|\left(B_{\alpha} A-I\right) B y\right\| \\
& =\left\|A_{\alpha} B y\right\|=\left\|\left(A_{\alpha}-P\right) B y\right\| \rightarrow 0 .
\end{aligned}
$$

$\left\{A_{\alpha}\right\}$ is said to be strongly ergodic if $D(P)=X$. In this case, we have $R(A)=A(D(A) \cap X)=A(D(A) \cap[N(A) \oplus \overline{R(A)}])=A(D(A) \cap \overline{R(A)})=D(B)$.

Conversely, the equality $D(B)=R(A)$ implies the strong ergodicity because, if not, there would be a $z \in D(A) \backslash D(P)$ and an $x \in D(A) \cap \overline{R(A)}$ such that $A z=\mathrm{Ax}$, which leads to $z=(z-x)+x \in N(A) \oplus \overline{R(A)}=D(P)$, a contradiction.

The purpose of this paper is to prove the following two uniform convergence theorems for the two systems $\left\{A_{\alpha}\right\}$ and $\left\{B_{\alpha}\right\}$. Applications to concrete examples are to be given in $\S 3$.

Theorem 1. Let $A$ be a densely defined closed linear operator in $X$, and let $\left\{A_{\alpha}\right\}$ and $\left\{B_{\alpha}\right\}$ be two nets in $B(X)$ which satisfy $(\mathrm{C} 1),(\mathrm{C} 2)$, and $(\mathrm{C} 3)$.

Then the following statements are equivalent:

(1) $\left\|A_{\alpha} \mid D(P)-P\right\| \rightarrow 0$,

(2) $D(P)=X$ and $\left\|A_{\alpha}-P\right\| \rightarrow 0$,

(3) $R(A)$ is closed,

(4) $R\left(A^{2}\right)$ is closed,

(5) $X=N(A) \oplus R(A)$,

(6) $\left\|B_{\alpha} \mid R(A)\right\|=O(1)$,

(7) $B$ is bounded and $\left\|B_{\alpha} \mid D(B)-B\right\| \rightarrow 0$.

Moreover, if (1)-(7) hold, then $D(B)=R\left(A^{2}\right)=R(A),\left\|A_{\alpha}-P\right\| \leq(M+1)$ $\times\left\|A A_{\alpha}\right\|\|B\|$ and $\left\|B_{\alpha} \mid D(B)-B\right\| \leq(M+1)\left\|A A_{\alpha}\right\|\|B\|^{2}$.

A Banach space $X$ is called a Grothendieck space if every weakly* convergent sequence in the dual space $X^{*}$ is weakly convergent, and is said to have the Dunford-Pettis property if $\left\langle x_{n}, x_{n}^{*}\right\rangle \rightarrow 0$ whenever $x_{n} \rightarrow 0$ weakly in $X$ and $x_{n}^{*} \rightarrow 0$ weakly in $X^{*}$. Among examples of Grothendieck spaces with the Dunford-Pettis property are $L^{\infty}, B(s, \Sigma), H^{\infty}(D)$, etc. (See [7].)

Theorem 2. Let $X$ be a Grothendieck space with the Dunford-Pettis property, and let $A,\left\{A_{\alpha}\right\}$, and $\left\{B_{\alpha}\right\}$ be as in Theorem 1. If $\left\{A_{\alpha}\right\}$ is strongly ergodic, then it is uniformly ergodic.

Thus, in this case the conditions (1)-(7) all are equivalent to each of $D(P)=$ $X$ and $D(B)=R(A)$. In view of the next theorem, we have another equivalent condition: $\overline{R\left(A^{*}\right)}=w^{*}-\operatorname{cl}\left(R\left(A^{*}\right)\right)$. 
Theorem 3. Let $X$ be a Grothendieck space, and let $A,\left\{A_{\alpha}\right\}$, and $\left\{B_{\alpha}\right\}$ be as in Theorem 1. Then $\left\{A_{\alpha}\right\}$ is strongly ergodic if and only if $\overline{R\left(A^{*}\right)}=w^{*}-$ $\operatorname{cl}\left(R\left(A^{*}\right)\right)$.

\section{Proof of Main Result}

Proof of Theorem 1. We prove the implication $s:(2) \Rightarrow(3) \Rightarrow(4) \Rightarrow(5) \Rightarrow(2)$, $(1) \Rightarrow(3) \Rightarrow(6) \Rightarrow(1)+(7)$.

(2) $\Rightarrow(3)$ (C3) implies that $\overline{R(A)}$ is invariant under $A_{\alpha}$ and $P A=0$, so that $\left\|A_{\alpha}\left|\overline{R(A)}\|=\|\left(A_{\alpha}-P\right)\right| \overline{R(A)}\right\| \leq\left\|A_{\alpha}-P\right\| \rightarrow 0$. Hence for some $\alpha,\left(A_{\alpha}-I\right) \mid \overline{R(A)}$ is invertible and so $\overline{R(A)} \subset R\left(A_{\alpha}-I\right) \subset R(A)$.

$(3) \Rightarrow(4)$. By the open mapping theorem (cf. [15, p. 213]), there is some $m>0$ such that each $x \in R(A)$ is equal to $A y$ for some $y \in D(A)$ with $\|y\| \leq$ $m\|x\|$. Hence $\left\|A_{\alpha} x\right\|=\left\|A A_{\alpha} y\right\| \leq\left\|A A_{\alpha}\right\| m\|x\|$, showing that $\left\|A_{\alpha} \mid R(A)\right\| \leq$ $m\left\|A A_{\alpha}\right\| \rightarrow 0$ and so $\left(A_{\alpha}-I\right) \mid R(A)$ is invertible for some $\alpha$. This, together with (C2) and (C3), implies that $R(A)=\left(A_{\alpha}-I\right) A D(A)=A\left(A_{\alpha}-I\right) D(A) \subset$ $A(D(A) \cap R(A))=R\left(A^{2}\right)$. Hence $R\left(A^{2}\right)=R(A)$ and is closed.

(4) $\Rightarrow$ (5). If $x \in D(A)$, then $\mathrm{Ax}=\lim \left\{A A_{\alpha} x-A\left(A_{\alpha}-I\right) x\right\}=$ $-\lim A\left(A_{\alpha}-I\right) x \in \overline{R\left(A^{2}\right)}=R\left(A^{2}\right)$. This shows that $R(A)=R\left(A^{2}\right)$ is closed and $D(A) \subset N(A)+R(A)$. Next, let $x \in X$, and let $\left\{x_{n}\right\}$ be a sequence in $D(A)$ such that $x_{n} \rightarrow x$. Then $A_{\alpha} x \in D(A)$ and $\left(A_{\alpha}-I\right) x=\lim _{n \rightarrow \infty}\left(A_{\alpha}-I\right) x_{n} \in$ $\overline{R(A)}=R(A)$ so that $x=A_{\alpha} x-\left(A_{\alpha}-I\right) x \in D(A)+R(A) \subset N(A)+R(A)$. Hence $X=N(A)+R(A)$. To see that this is a direct sum, let $x \in N(A) \cap R(A)$. Then there is a $y$ such that $A y=x \in N(A) \subset N\left(A_{\alpha}-I\right)$ for all $\alpha$. But then $x=A_{\alpha} x=A_{\alpha} A y \rightarrow 0$.

$(5) \Rightarrow(2)$. The closedness of $A$ and assumption (5) imply $R(A)$ is closed (see [16, p. 217]). Then, as was shown in $(3) \Rightarrow(4)$, we have $\left\|\left(A_{\alpha}-P\right) \mid R(A)\right\|=$ $\left\|A_{\alpha} \mid R(A)\right\| \leq m\left\|A A_{\alpha}\right\| \rightarrow 0$. Because $\left(A_{\alpha}-P\right) \mid N(A)=0$, it follows that $\left\|A_{\alpha}-P\right\| \rightarrow 0$.

(1) $\Rightarrow(3)$. Since $A_{\alpha} A \subset A A_{\alpha}$, the space $D(P)=N(A) \oplus \overline{R(A)}$ is invariant under $A_{\alpha}$, and $A \mid D(P)$ is a densely defined closed operator in $D(P)$. Applying (2) $\Rightarrow(5)$ to $\left\{A_{\alpha} \mid D(P)\right\}$ and $A \mid D(P)$, we infer that $D(P)=N(A \mid D(P)) \oplus$ $R(A \mid D(P))$. Since $N(A \mid D(P))=N(A)$ and $R(A \mid D(P)) \subset R(A)$, it follows from the two expressions of $D(P)$ that $\overline{R(A)}=R(A \mid D(P))$ and hence $\overline{R(A)}=$ $R(A)$.

(3) $\Rightarrow(6)$. If $R(A)$ is closed, then as shown in (3) $\Rightarrow(4)$, we have $R\left(A^{2}\right)=$ $R(A)$, so that $D(B)=A(D(A) \cap R(A))=R\left(A^{2}\right)=R(A)$ is closed. Since $B_{\alpha} y \rightarrow B y$ for all $y \in D(B)$, the uniform boundedness principle implies (6).

(6) $\Rightarrow(1)+(7)$. (6) implies that $B$ is bounded and hence $R(A \mid D(P))=$ $A(D(A) \cap \overline{R(A)})=D(B)$ is closed. An application of $(3) \Rightarrow(2)$ to $\left\{A_{\alpha} \mid D(P)\right\}$ asserts that $\left\|A_{\alpha} \mid D(P)-P\right\| \rightarrow 0$, from which, together with $(*)$, we see that $\left\|B_{\alpha}\left|D(B)-B\|\leq\| A_{\alpha}\right| D(P)-P\right\|\|B\| \rightarrow 0$.

Finally, if (1)-(7) hold, then for any $x \in X$, we have $x-P x \in R(A)=$ $R\left(A^{2}\right)=D(B)$ so that $A B(x-P x)=x-P x$ and

$$
\left\|A_{\alpha} x-P x\right\|=\left\|A_{\alpha}(x-P x)\right\|=\left\|A_{\alpha} A B(I-P) x\right\| \leq\left\|A A_{\alpha}\right\|\|B\|(M+1)\|x\| .
$$

Hence $\left\|A_{\alpha}-P\right\| \leq(M+1)\left\|A A_{\alpha}\right\|\|B\|$ and $\left\|B_{\alpha} \mid D(B)-B\right\| \leq(M+1)\left\|A A_{\alpha}\right\|\|B\|^{2}$.

To prove Theorems 2 and 3 we need the following lemma. 
Lemma. If $A,\left\{A_{\alpha}\right\}$, and $\left\{B_{\alpha}\right\}$ satisfy conditions (C1), (C2), and (C3), then $A^{*},\left\{A_{\alpha}^{*}\right\}$, and $\left\{B_{\alpha}^{*}\right\}$ also satisfy these conditions.

Proof. It suffices to show that if $E \in B(X)$ is such that $R(E) \subset D(A)$ and $E A \subset A E$, then $R\left(E^{*}\right) \subset D\left(A^{*}\right)$ and $E^{*} A^{*} \subset A^{*} E^{*}=(A E)^{*}$. If $x^{*} \in D\left(A^{*}\right)$, then $\left\langle\mathrm{Ax}, E^{*} x^{*}\right\rangle=\left\langle E \mathrm{Ax}, x^{*}\right\rangle=\left\langle x, E^{*} A^{*} x^{*}\right\rangle$ for all $x \in D(A)$, so that $E^{*} x^{*} \in D\left(A^{*}\right)$ and $A^{*} E^{*} x^{*}=E^{*} A^{*} x^{*}=(A E)^{*} x^{*}$. Hence $E^{*} A^{*} \subset A^{*} E^{*}$. To show $R\left(E^{*}\right) \subset D\left(A^{*}\right)$ and $A^{*} E^{*}=(A E)^{*}$, we use the fact that $A^{*}$ is weakly* densely defined and $w^{*}-w^{*}$-closed. For any $x^{*} \in X^{*}$, let $\left\{x_{\alpha}^{*}\right\}$ be a net in $D\left(A^{*}\right)$ such that $x_{\alpha}^{*} \rightarrow x^{*}$ weakly*. Then $E^{*} x_{\alpha}^{*}$ and $A^{*} E^{*} x_{\alpha}^{*}$ $\left(=(A E)^{*} x_{\alpha}^{*}\right)$ converges weakly* to $E^{*} x^{*}$ and $(A E)^{*} x^{*}$, respectively. This implies that $E^{*} x^{*} \in D\left(A^{*}\right)$ and $A^{*} E^{*} x^{*}=(A E)^{*} x^{*}$.

Proof of Theorem 2. Since $A_{\alpha} \mid R(P)=I$, we may assume $A_{\alpha} \rightarrow 0$ strongly and show that $\left\|A_{\alpha}\right\| \rightarrow 0$, without loss of generality. Take a sequence $A_{n} \equiv$ $A_{\alpha_{n}} \rightarrow 0$. Then $A_{n} x$ converges strongly to 0 for all $x \in X$. This implies that $\left\{A_{n}^{*} x_{n}^{*}\right\}$ converges weakly* and hence weakly to 0 whenever $\left\{x_{n}^{*}\right\}$ is bounded. In particular, $\left\{A_{n}^{*} x^{*}\right\} \rightarrow 0$ weakly for all $x^{*} \in X^{*}$. The convergence actually holds in the strong topology, by the strong ergodic theorem (applied to $\left\{A_{n}^{*}\right\}$ ). This fact in turn implies that $\left\{A_{n} x_{n}\right\}$ converges weakly to 0 whenever $\left\{x_{n}\right\}$ is bounded. Now, it follows from a lemma of Lotz [7] that $\left\|A_{n}^{2}\right\| \rightarrow 0$. Thus $I-A_{m}$ is invertible for a sufficiently large $m$. By (C2) and (C3) we obtain that

$$
\begin{aligned}
\left\|A_{n}\right\| & =\left\|A_{n}\left(I-A_{m}\right)\left(I-A_{m}\right)^{-1}\right\|=\left\|A_{n} A B_{m}\left(I-A_{m}\right)^{-1}\right\| \\
& \leq\left\|A A_{n}\right\|\left\|B_{m}\right\|\left\|\left(I-A_{m}\right)^{-1}\right\| \rightarrow 0 \quad \text { as } n \rightarrow \infty .
\end{aligned}
$$

Application of Theorem 1 to $\left\{A_{n}\right\}$ and $\left\{A_{\alpha}\right\}$ shows first that $R(A)$ is closed and then that $\left\|A_{\alpha}\right\| \rightarrow 0$.

Proof of Theorem 3. If $D(P)=X$, then for every $x^{*} \in X^{*}$ we have

$$
w-\lim _{n \rightarrow \infty} A_{\alpha_{n}}^{*} x^{*}=w^{*}-\lim _{n \rightarrow \infty} A_{\alpha_{n}}^{*} x^{*}=P^{*} x^{*},
$$

where $\left\{A_{\alpha_{n}}\right\}$ is any subsequence of $\left\{A_{\alpha}\right\}$ The strong ergodic theorem applied to $\left\{A_{\alpha_{n}}^{*}\right\}$, shows that $* P^{*} x^{*}=s-\lim _{n \rightarrow \infty} A_{\alpha_{n}}^{*} x^{*}$ for all $x^{*} \in X^{*}, N\left(P^{*}\right)=\overline{R\left(A^{*}\right)}$. Hence $\overline{R\left(A^{*}\right)}=R(P)^{\perp}=N(A)^{\perp}=\left[{ }^{\perp} R\left(A^{*}\right)\right]^{\perp}=w^{*}$ - $\operatorname{cl}\left(R\left(A^{*}\right)\right)$.

Conversely, if $\overline{R\left(A^{*}\right)}=w^{*}-\mathrm{cl}\left(R\left(A^{*}\right)\right)$, then $D(P)^{\perp}=\{N(A) \oplus \overline{R(A)}\}^{\perp}=$ $\left[{ }^{\perp} R\left(A^{*}\right)\right]^{\perp} \cap \overline{R(A)}^{\perp}=w^{*}-\mathrm{cl}\left(R\left(A^{*}\right)\right) \cap N\left(A^{*}\right)=\overline{R\left(A^{*}\right)} \cap N\left(A^{*}\right)=\{0\}$, again following from the strong ergodic theorem applied to $\left\{A_{\alpha}^{*}\right\}$. Since $D(P)$ is closed, it must be equal to $X$.

\section{EXAMPLES}

We consider applications to $n$-times integrated semigroups, $(Y)$-semigroups, and cosine operator functions.

3.1. $n$-times integrated semigroups. Let $n$ be a positive integer. A strongly continuous family $\{T(t) ; t \geq 0\}$ in $B(X)$ is called an $n$-times integrated semigroup (see $[1,15])$ if $T(0)=I$ and

$$
\begin{array}{r}
T(t) T(s)=\frac{1}{(n-1) !}\left(\int_{t}^{t+s}(t+s-r)^{n-1} T(r) d r-\int_{0}^{s}(t+s-r)^{n-1} T(r) d r\right) \\
(s, t \geq 0) .
\end{array}
$$


A $C_{0}$-semigroup is called an $o$-times integrated semigroup. It is known that the integrals over $[0, t], t \geq 0$, of an $n$-times $(n \geq 0)$ integrated semigroup form an $(n+1)$-times integrated semigroup, but not conversely.

$T(\cdot)$ is called nondegenerate if $T(t) x=0$ for all $t>0$ implies $x=0$. It is called exponentially bounded if there are $M \geq 0, w \in R$ such that $\|T(t)\| \leq$ $M e^{w t}$ for all $t \geq 0$. If $T(\cdot)$ is nondegenerate and exponentially bounded, then there exists a unique closed operator $A$ satisfying $(w, \infty) \subset \rho(A)$ and $(\lambda-A)^{-1} x=\int_{0}^{\infty} \lambda^{n} e^{-\lambda t} T(t) d t$ for $x \in X$ and $\lambda>w$. This operator is called the generator of $T(\cdot)$. It is not necessarily densely defined. We only consider the case when $A$ is densely defined. This includes all $C_{0}$-semigroups.

It is known [1, Proposition 3.3] that $\int_{0}^{t} T(s) x d s \in D(A)$ and $A \int_{0}^{t} T(s) x d s=$ $T(t) x=\left(t^{n} / n !\right) x$ for all $x \in X$, and $\int_{0}^{t} T(s) A x d s=T(t) x-\left(t^{n} / n !\right) x$ for all $x \in D(A)$. Since $A$ is closed, taking integration gives that

$$
\int_{0}^{t} T(s) x d s-\left(t^{n+1} /(n+1) !\right) x= \begin{cases}A \int_{0}^{t} \int_{0}^{s} T(u) d d u d s & \text { for } x \in X, \\ \int_{0}^{t} \int_{0}^{s} T(u) \mathrm{Ax} d u d s & \text { for } x \in D(A) .\end{cases}
$$

Let $A_{t}:=(n+1) ! t^{-n-1} \int_{0}^{t} T(s) d s$ and $B_{t}:=-(n+1) ! t^{-n-1} \int_{0}^{t} \int_{0}^{s} T(u) d u d s$ for $t>0$. Then $B_{t} A \subset A B_{t}=I-A_{t}$ and $A_{t} A \subset A A_{t}=(n+1) ! T(t) / t^{n+1}-(n+1) I / t$. Suppose $\|T(t)\|=O\left(t^{n}\right)(t \rightarrow \infty)$. Then $A,\left\{A_{t}\right\}$, and $\left\{B_{t}\right\}$ satisfy (C1), (C2), and (C3) as $t \rightarrow \infty$. On the other hand, the systems $\left\{\lambda(\lambda-A)^{-1}\right\}$, $\left\{-(\lambda-A)^{-1}\right\}$ clearly satisfy $(\mathrm{C} 1),(\mathrm{C} 2)$, and (C3) as $\lambda \rightarrow 0$ too. Hence the strong ergodic theorem and the theorems in $\S 1$ are applicable to $\left\{A_{t}\right\}$ with $\left\{B_{t}\right\}$ and $\left\{\lambda(\lambda-A)^{-1}\right\}$ with $\left\{-(\lambda-A)^{-1}\right\}$, and the next two theorems follow immediately.

Theorem 4. Let $\{T(t) ; t \geq 0\}$ be a nondegenerate $n$-times integrated semigroup with generator $A$ densely defined. Suppose $\|T(t)\|=O\left(t^{n}\right) \quad(t \rightarrow \infty)$. Let $A_{t}$ and $B_{t}$ be as previously defined. Then $s-\lim _{t \rightarrow \infty} A_{t} x$ and $s-\lim _{\lambda \rightarrow 0^{+}} \lambda(\lambda-A)^{-1} x$ exist and are equal if one of them exists, and the limits define a bounded linear projection $P$ onto $N(A)$ along $\overline{R(A)}$. For $y \in \overline{R(A)}, s-\lim _{t \rightarrow \infty} B_{t} y$ and $s-\lim _{\lambda \rightarrow 0^{+}}(A-\lambda)^{-1} y$ exist and are equal if one of them exists, and the limits define an operator $B$ which sends each $y \in A(D(A) \cap \overline{R(A)})$ to the unique solution $x=B y$ of $\mathrm{Ax}=y$ in $\overline{R(A)}$.

Theorem 5. Under the hypothesis of Theorem 4, the following statements are equivalent:

(1) $\left\|A_{t}-P\right\| \rightarrow 0$ as $t \rightarrow \infty$,

(2) $\left\|\lambda(\lambda-A)^{-1}-P\right\| \rightarrow 0$ as $t \rightarrow 0^{+}$,

(3) $R(A)$ is closed,

(4) $\left\|B_{t} \mid R(A)\right\|=O(1) \quad(t \rightarrow \infty)$,

(5) $\left\|B_{t} \mid R(A)-B\right\| \rightarrow 0$ as $t \rightarrow \infty$

(6) $\left\|(\lambda-A)^{-1} \mid R(A)-B\right\| \rightarrow 0$ as $\lambda \rightarrow 0^{+}$.

Moreover, when $X$ is a Grothendieck space with the Dunford-Pettis property, $D(P)=X$ and $\overline{R\left(A^{*}\right)}=w^{*}-\operatorname{cl}\left(R\left(A^{*}\right)\right)$ are two more equivalent conditions.

Remarks. (i) When (1)-(6) hold, we have $\left\|A_{t}-P\right\|=O(1 / t),\left\|B_{t} \mid R(A)-B\right\|=$ $O(1 / t) \quad(t \rightarrow \infty)$, and $\left\|\lambda(\lambda-A)^{-1}-P\right\|=O(\lambda),\left\|(\lambda-A)^{-1} \mid R(A)-B\right\|=O(\lambda)$, $\lambda \rightarrow 0^{+}$. 
(ii) In the case $n=0$, Theorem 4 is well known (see [3, pp. 58-60] for the first part, and [4] for the second part), the equivalence of (1), (2), and (3) in Theorem 5 is proved in [6] (see also [10]), the equivalence of strong ergodicity and $\left.\overline{R\left(A^{*}\right)}=w^{*}-\operatorname{cl}\left(R A^{*}\right)\right)$ in a Grothendieck space is proved in [9], and the equivalence of strong ergodicity and uniform ergodicity in a Grothendieck space with the Dunford-Pettis property is proved in [7]. The theorems with $n \geq 1$ are new.

3.2. ( $Y$ )-semigroups. Let $Y$ be a closed subspace of $X^{*}$ such that the canonical imbedding of $X$ into $Y^{*}$ is isometric. A semigroup $\{T(t) ; t \geq 0\}$ of operators on $X$ is called a $(Y)$-semigroup (cf. $[8,11]$ ) if $Y$ is invariant under $T^{*}(t)$ for all $t \geq 0$ and $T(\cdot) x$ is $\sigma(X, Y)$-continuous on $[0, \infty)$ and locally $\sigma(X, Y)$-Pettis integrable for all $x \in X$. The generator $A$ of $T(\cdot)$ is defined by Ax $:=\sigma(X, Y)-\lim _{t \rightarrow 0^{+}} t^{-1}(T(t)-I) x$. A $C_{0}$-semigroup on $X$ is a $\left(X^{*}\right)$-semigroup, and its dual semigroup is a $(X)$-semigroup. The tensor product $T(t)$ of two $C_{0}$-semigroups $e^{t A}$ and $e^{-t B}$ on $X$ is a $(Y)$-semigroup on $B(X)$ for some suitable subspace $Y$ of $B(X)^{*}$; its generator is the operator $\Delta: C \rightarrow A C-C B$.

The strong convergence of ergodic limits of a $(Y)$-semigroup and that of approximate solutions of the corresponding equation $\mathrm{Ax}=y$ have been discussed in [13, Example VI]. The result is the same as Theorem 4 with $n=0$. By applying Theorems 1 and 2 one can easily see that Theorem 5 with $n=0$ holds for $(Y)$-semigroups too. Since $S(t):=\int_{0}^{t} T(s) d s, t \geq 0$, forms a onceintegrated semigroup, we can apply Theorems 4 and 5 to $S(\cdot)$ to obtain ergodic theorems for $(C, 2)$-means of $T(\cdot)$; they are Theorems 4 and 5 with $A_{t}=2 t^{-2} \int_{0}^{t} \int_{0}^{s} T(u) d u d s$ and $B_{t}=-2 t^{-2} \int_{0}^{t} \int_{0}^{s} \int_{0}^{u} T(v) d v d s$.

3.3. Cosine operator functions. A strongly continuous family $\{C(t) ; t \in R\}$ in $B(X)$ is called a cosine operator function if $C(0)=I$ and $C(t+s)+C(t-s)=$ $2 C(t) C(s), s, t \in R$. The generator $A$, defined by $\mathrm{Ax}:=C^{\prime \prime}(0) x$, is a densely defined closed operator.

For $t>0$ let

$$
A_{t}:=2 t^{-2} \int_{0}^{t} \int_{0}^{s} C(u) d u d s
$$

and

$$
B_{t}=-2 t^{-2} \int_{0}^{t} \int_{0}^{s} \int_{0}^{u} \int_{0}^{v} C(w) d w d v d u d s .
$$

Then we have $B_{t} A \subset A B_{t} \subset I_{t}-A$ and $A_{t} A \subset A A_{t}=2 t^{-2}(C(t)-I)$. The strong convergence of $A_{t} x$ and $B_{t} y$ as $t \rightarrow \infty$ has been discussed in [13, Example VII]. We now deduce from Theorems 1 and 2 the following theorem about uniform convergence.

Theorem 6. Suppose that $\left\|\int_{0}^{t} \int_{0}^{s} C(u) d u d s\right\|=O\left(t^{2}\right) \quad(t \rightarrow \infty)$ and $\|C(t)\|=$ $o\left(t^{2}\right) \quad(t \rightarrow \infty)$. Then, with $A_{t}$ and $B_{t}$ defined as above, the conclusion of Theorem 5 remains valid.

\section{CONCLUDING REMARK}

Our Theorems 1, 2, and 3 can also be used to deduce uniform ergodic theorems for discrete semigroups (cf. [5,7]) and uniform ergodic theorems for pseudoresolvents (cf. [10,12]). 


\section{REFERENCES}

1. W. Arendt, Vector valued Laplace transforms and Cauchy problems, Israel J. Math. 59 (1987), 327-352.

2. W. G. Dotson, Jr., An application of ergodic theory to the solution of linear functional equations in Banach spaces, Bull. Amer. Math. Soc. 75 (1969), 347-352.

3. J. A. Goldstein, Semigroups of linear operators and applications, Oxford, Univ. Press, New York, 1985.

4. U. Krengel and M. Lin, On the range of the generator of a Markovian semigroup, Math. Z. 185 (1984), 553-565.

5. M. Lin, On the uniform ergodic theorem, Proc. Amer. Math. Soc. 43 (1974), 337-340.

6. __ On the uniform ergodic theorem. II, Proc. Amer. Math. Soc. 46 (1974), 217-225.

7. H. P. Lotz, Tauberian theorems for operators on $L^{\infty}$ and similar spaces, Functional Analysis: Surveys and Recent Results III, North-Holland, Amsterdam, 1984, pp. 117-133.

8. S.-Y. Shaw, Ergodic properties of operator semigroups in general weak topologies, J. Funct. Anal. 49 (1982), 152-169.

9. ___ Ergodic theorems for semigroups of operators on a Grothendieck space, Proc. Japan Acad. Ser. A. Math. Sci. 59 (1983), 132-135.

10. __ Uniform ergodic theorems for locally integrable semigroups and pseudoresolvents, Proc. Amer. Math. Soc. 98 (1986), 61-67.

11. S.-Y. Shaw and S. C. Lin, On the equations $\mathrm{Ax}=q$ and $S X-X T=Q$, J. Funct. Anal. 77 (1988), 352-363.

12. S.-Y. Shaw, Asymptotic behavior of pseudoresolvents on some Grothendieck spaces, Publ. Res. Inst. Math. Sci. 24 (1988), 277-282.

13. __ Mean ergodic theorems and linear functional equations, J. Funct. Anal. 87 (1989), 428-441.

14. _ Solvability of linear functional equations in Lebesgue spaces, Publ. Res. Inst. Math. Sci. 26 (1990), 691-699.

15. N. Tanaka and I. Miyadera, Some remarks on C-semigroups and integrated semigroups, Proc. Japan Acad. Ser. A. Math. Sci. 63 (1987), 139-142.

16. A. E. Taylor and D. C. Lay, Introduction to functional analysis, 2nd ed., Wiley, New York, 1980.

Department of Mathematics, National Central University, Chung-Li, Taiwan, RePUBLIC OF CHINA 